\title{
An Innovative Non-Pharmacologic Treatment for Delusional Misidentification in Persons with Major Neurocognitive Disorder
}

Anne E. Adams, PhD $;{ }^{1}$ Jennifer Rittereiser, MPH $;{ }^{2}$ Alisha Garcia, MS, LPC, NCC $;{ }^{3}$ Melany Sattler, MSW; ${ }^{4}$ James M. Ellison, MD MPH ${ }^{5}$

1. Managing Director, Applied Research, SimpleC LLC

2. Director, Swank Memory Ambassadors Program, Memory Care and Geriatrics, ChristianaCare

3. Psychotherapist, The Anxiety and Stress Management Institute; Former Director, Clinical Mental Health Services, SimpleC LLC

4. Vice President, Clinical Services, SimpleC LLC

5. Swank Foundation Endowed Chair, Memory Care and Geriatrics, ChristianaCare; Professor, Psychiatry and Human Behavior, Sidney Kimmel Medical College, Thomas Jefferson University; Editor-in-Chief, Journal of Geriatric Psychiatry and Neurology

\begin{abstract}
Misidentification delusions are false, fixed beliefs that assign an incorrect identity to a previously familiar or unfamiliar person or place. Such delusions are common in several neuropsychiatric disorders and place a particular burden on individuals with Major Neurocognitive Disorder and their caregivers. No standard pharmacologic or non-pharmacologic treatment approaches have been shown to be consistently effective in addressing this problem. We describe two caregivercare recipient dyads in which an innovative non-pharmacologic, digital intervention reduced delusional misidentification, improved care recipient behavior, and decreased caregiver burden.
\end{abstract}

\section{Background}

Delusional Misidentification (DM) is among the most disruptive and recalcitrant behavioral symptoms encountered in the care of persons with Major Neurocognitive Disorders (MaND). More disruptive than simple loss of recognition, delusional beliefs in a caregiver-care recipient dyad can seriously exacerbate caregiver burden while undermining the effectiveness and safety of care. ${ }^{1}$

There is no specific diagnostic code for DM in the DSM-5, but clinically significant delusions in a person with dementia justify the label of MaND "with behavioral disturbance". ${ }^{2}$ Several syndromes of DM occur in the context of MaND:

- In Capgras syndrome, a familiar person is incorrectly identified as an imposter.

- In reduplicative paramnesia, a familiar person or place exists in multiple, different versions.

- In mirror self-misidentification, one's reflection is experienced as a separate person.

- In Fregoli syndrome, a stranger is mistakenly identified as a disguised familiar person. 
Prevalence estimates of DM vary widely, between $5 \%$ and $82 \%{ }^{3}$ The most common types are Capgras syndrome and reduplicative paramnesia. DM may be especially common in Dementia with Lewy Bodies, Vascular Dementia, and Alzheimer's disease. ${ }^{2}$ When DM is present, affected individuals and their caregivers must manage, often unsuccessfully, the challenging consequences of a disrupted alliance. These can range from uncooperativeness and even aggressive resistance to inappropriate familiarity. ${ }^{1}$ When DM develops within a caregiving dyad, the care of an affected person becomes enormously more complicated.

The pathophysiology of DM syndromes may involve an interactive effect of lesions affecting the fusiform gyrus, which mediates facial recognition; the perirhinal cortex and right hemisphere, which mediate familiarity; and the frontal lobes, which test reality. ${ }^{4}$ Neurologically, DM appears like a "disconnection syndrome" in which the links between recognition, emotional response, and reality testing go awry.

No medication or non-pharmacologic intervention has been shown to be effective in consistently reducing DM. However, evidence supports the effectiveness of non-pharmacologic approaches such as reminiscence therapy, music therapy, validation, and simulated presence in mitigating the severity of various non-cognitive symptoms associated with dementia. ${ }^{5}$ Digital interventions are emerging as an effective means for delivering care. Research has shown successful reduction of non-cognitive symptoms in people with MaND by means of personalized digital interventions delivered in a formal care setting. ${ }^{6}$ For caregiver-recipient dyads living at home, too, personalized digital interventions have proven successful in reducing non-cognitive symptoms of MaND, facilitating positive and meaningful engagement, and providing overall caregiver support reduced burden. ${ }^{7-9}$

We report here the successful treatment of DM in two caregiver-recipient dyads using a digital tool that was modified to achieve two objectives important in the non-pharmacologic care of people with MaND and DM: (1) Induce a positive affective state through reminiscence therapy and curated, personalized music; and (2) reinforce recognition memory through spaced repetition and consistency of visual cues linked with positive reminiscence, as is used in cognitive rehabilitation approaches, thereby improving feelings of familiarity and enhancing a positive emotional response towards the caregiver.

\section{Case Presentations}

\section{Case Vignette - Dyad \#1}

Mr. X, a 78-year-old, married, non-Hispanic White male, was residing at home with his wife and grandson at the time of referral to a Memory Disorders Clinic (MDC) for evaluation of an episode of disorientation. His wife described his year-long history of cognitive and behavioral symptoms including memory impairment, disorientation to date and location, restlessness and pacing, and suspected hallucinations. His activities of daily living were partially dependent, and he needed assistance with his instrumental activities of daily living. His Mini-Mental State

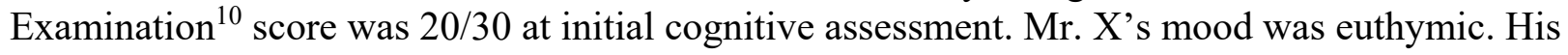
medical history was notable for vascular risk factors: previous myocardial infarction, adult-onset diabetes mellitus, hyperlipidemia, and hypertension. He denied alcohol use. Routine laboratory study results were within normal ranges. Brain MRI showed mild to moderate parenchymal volume loss with significant patchy areas of T2/FLAIR hyperintensity in the periventricular, subcortical, and deep white matter. His presentation was consistent with a diagnosis of vascular 
major neurocognitive disorder. During the year after his initial referral, Mr. X's behavioral symptoms became very disruptive to continued home care. His sundowning and agitation required intervention from law enforcement personnel. Behavioral interventions, supplemented with quetiapine $50 \mathrm{mg}$ at bedtime, failed to halt the increasingly frequent delusional belief that his wife was an imposter. His DM lasted for hours at a time. His behavior, when delusional, made it necessary for his wife to leave the home repeatedly. Multiple additional medication trials including escitalopram up to $10 \mathrm{mg} / \mathrm{d}$, donepezil up to $23 \mathrm{mg} / \mathrm{d}$, buspirone up to $5 \mathrm{mg}$ twice daily, risperidone up to $0.25 \mathrm{mg}$ three times daily, melatonin up to $10 \mathrm{mg}$ at bedtime and cannabidiol oil failed to affect his delusion or improve his behavior.

\section{Case Vignette - Dyad \#2}

Mrs. Y, an 89-year-old, married, non-Hispanic White female, was residing in an independent living community apartment with her husband at the time of her referral to the MDC. She expressed a fear that she was losing her memory. Her husband reported that her memory decline had become apparent two years earlier, after an episode of complete heart block which included a 14-hour run of bradycardia with a pulse of 25 beats per minute, culminating in insertion of a pacemaker. Mr. Y reported that his wife's cognitive symptoms included forgetting names, losing her train of thought, and trouble with "getting her words out." She experienced anxiety, crying spells, and had a history of depressive episodes. She reported "seeing" her deceased parents. Her confusion regularly increased in the evenings. Her activities of daily living remained independent. Her instrumental activities were partially intact, though bill-paying required assistance. She routinely participated in a sitting exercise class. She scored 16/30 on the Montreal Cognitive Assessment (MoCA) ${ }^{11}$ and 10/15 on the Geriatric Depression Scale. ${ }^{12}$ Mrs. Y's medical history included multiple falls, a right parietal subdural hematoma requiring craniotomy, treated hypothyroidism, complete heart block treated with a pacemaker, diminished auditory acuity, and nocturia which interrupted her sleep 4 to 5 times nightly. Her head CT scan showed evidence of periventricular small vessel disease, a small lacunar infarct in the left basis pontis, and a small chronic left cerebellar infarct. In her delusional thinking, Mrs. Y split her husband into two separate men with the same name, one trustworthy and the other a "menace." Her psychiatrist had prescribed quetiapine $12.5 \mathrm{mg}$ twice daily and mirtazapine $7.5 \mathrm{mg}$ at bedtime for her delusions, with limited benefit.

\section{Treatment Procedure}

Mr. X, Mrs. Y, and their caregiving spouses each gave written informed consent to participate in an IRB-approved clinical trial testing a non-pharmacological intervention designed for the treatment of DM. The intervention began with a two-week assessment of baseline functioning and behavior. During this time, images of each person with MaND and their spouse were collected with the goal of obtaining at least 50 photographs. These images were assessed for familiarity (five point scale), valence of the emotional response (positive, neutral, negative), and recognition using a facial recognition assessment developed for this study. Based on the results of the initial assessment, a total of 35 images were selected for each dyad's intervention program. A personalized digital intervention using the selected images and individualized music was created on the SimpleC Platform for each person with MaND and accessed via the Companion application on an iPad tablet. 
During the study, three active phases alternated with three control phases. Each phase lasted for two weeks except for the final control phase, which lasted four weeks. During the study's active phases, the dyad engaged with their personalized intervention. During the control phases, the dyad engaged with neutral images such as landscapes. The intervention consisted of two daily 15-minute sessions during which the dyad viewed and reengaged with the digital therapeutic programs. The investigators conducted weekly check-ins throughout the study and one week after a final, four week control phase. Each dyad's caregiving spouse kept a daily diary and symptom log throughout the trial.

Investigator-administered outcome measurements were obtained using the Neuropsychiatric Inventory (NPI), ${ }^{13}$ the Misidentification Delusion Questionnaire (MDQ), ${ }^{14}$ the Zarit Caregiver Burden Scale, (Zarit), ${ }^{15}$ the Quality of Life in Alzheimer's Disease Scales for both person with MaND and their spouse (QoL-AD), ${ }^{16}$ as well as expectation \& experience questionnaires developed for this study.

Facial recognition for all collected images was assessed three times as described above. The procedure also included intermediate assessments of some images, at the end of each active and control phase during the intervention to help engage deeper processing for better retention. ${ }^{17}$

\section{Outcomes}

\section{Dyad \#1}

Mr. X showed improvements in neuropsychiatric symptoms (NPI) and quality of life over the intervention period (Table 1). Specific NPI improvements were noted for symptoms of delusion (drop from 9 to 1), hallucination (drop from 8 to 3), and agitation (drop from 6 to 2) through a reduction in frequency, severity, and stressfulness of the symptom.

The MDQ also improved as well. The change reflected improved home recognition. The MDQ did not capture wife recognition, but the diary did. At the end of the intervention, Mr. X agreed that the intervention had helped him to be more connected, that he will know others better, and others will know him better. He wanted to keep the intervention, saying "it is something very nice" and "good for you." At the end of the one month final control phase - without the intervention - Mr. X did not mention these benefits.

Table 1: Standard Measures for Dyad \#1

\begin{tabular}{lcccc}
\hline Measure & $\begin{array}{c}\text { Session 1 } \\
\text { (Baseline) }\end{array}$ & $\begin{array}{c}\text { Session 9 } \\
\text { (End of } \\
\text { intervention) }\end{array}$ & $\begin{array}{c}\text { Session 10 } \\
\text { (End of final control } \\
\text { phase) }\end{array}$ & Max \\
\hline $\begin{array}{l}\text { Lower scores are better } \\
\text { NPI Total (10 items) }\end{array}$ & 55 & 44 & 60 & 120 \\
NPI Stress (10 items) & 14 & 12 & 19 & 50 \\
MDQ & 2 & 0 & 1 & 11 \\
Zarit & 26 & 38 & 37 & 88 \\
Higher scores are better & & 39 & 37 & 52 \\
QoL-AD - CR & 32 & 39 & 22 & 52 \\
QoL-AD - CG & 28 & 23 &
\end{tabular}

In the experience questionnaire, Mrs. X reported that the intervention helped her husband to recognize her more often, stating that he "became more affectionate" after using it. She liked the 
"time spent together reminiscing" with "nothing distracting our attention." She reported that she was "thrilled that he was more passionate," and that it "stimulated conversation." Mrs. X reported more overall caregiver stress as measured by the Zarit and QoL-AD, but less stress related to the neuropsychiatric symptoms as measured by the NPI. The caregiver's diary documented the decreasing frequency of DM over the course of the study.

Here are instances of non-recognition quoted from the diary to illustrate how DM manifested itself in the life of Mr. and Mrs. X.

- Looking at pictures. Didn't recognize me in several.

- Entering the mall, he told me "[Mrs. X] and I did this lots of times" - I just said "really."

- Getting ready to go to a birthday party gathering. [Mr. X] asked, "Where is [Mrs. $X ?]$ "

- Watching TV, jumped up (where's [Mrs. X]), left the room, went upstairs. Came back, knew me and was fine. She had been with our daughter.

- Where's [Mrs. X]? I asked, "what does she look like?” He answered, "I don't know what you're talking about."

- He asked, “Where's [Mrs. X]”? I asked if she was young or old. He answered, “don't know." What does she look like-don't know anything. That was it.

\section{Dyad \#2}

Mrs. Y showed improvements in neuropsychiatric symptoms (NPI) and MDQ during the intervention period (Table 2). Mr. Y exhibited reduced caregiver stress related to neuropsychiatric symptoms (NPI) and stable caregiver burden as measured by Zarit and QoLAD.

Table 2: Standard Measures for Dyad \#2

\begin{tabular}{|c|c|c|c|c|}
\hline Measure & $\begin{array}{l}\text { Session 1 } \\
\text { (Baseline) }\end{array}$ & $\begin{array}{l}\text { Session 9 } \\
\text { (End of } \\
\text { intervention) }\end{array}$ & $\begin{array}{c}\text { Session } 10 \\
\text { (End of final control } \\
\text { phase) }\end{array}$ & Max \\
\hline \multicolumn{5}{|l|}{ Lower scores are better } \\
\hline NPI Total (10 items) & 22 & 16 & 14 & 120 \\
\hline NPI Stress (10 items) & 12 & 6 & 10 & 50 \\
\hline MDQ & 4 & 1 & 2 & 11 \\
\hline Zarit & 19 & 20 & 23 & 88 \\
\hline \multicolumn{5}{|l|}{ Higher scores are better } \\
\hline QoL-AD - CR & 34 & 24 & 39 & 52 \\
\hline QoL-AD - CG & 33 & 33 & 30 & 52 \\
\hline
\end{tabular}

Of particular interest is the improvement captured by the facial recognition assessment. At baseline, Mrs. Y rated $84 \%$ of the images as very familiar and responded negatively to $8 \%$ of the images and neutrally to $10 \%$ of them. At the end of the intervention period, ratings increased to $100 \%$ familiarity and $100 \%$ positive valence. Familiarity remained high at the end of the final control phase, with only a slight decrease in emotional valence. 
In the experience questionnaire, the caregiver Mr. Y noted "improved closeness when using pictures and music," and an overall more positive feeling. He stated that the intervention helped his wife recognize him more often and more quickly. These benefits were more prominent at the end of the intervention than the final one-month control phase without intervention. Mr. Y noted that the intervention enhanced their relationship and helped him enjoy life with his wife.

Here are instances of non-recognition quoted from the diary to illustrate how DM manifested itself in the life of Mr. and Mrs. Y.

- A very good day. Greeted each other with a kiss this morning. Daughter came over in the morning to help organize [Mrs. Y's] closest. Very pleasant visit. Normal afternoon nap for [Mrs. Y], and then dinner with friends in dining room. Short episode of confusion this morning.

- [Mrs. Y] was in MI [misidentification] mode much of the day. I was seen as a good friend. She had an hour or so with mental confusion. I got frustrated trying to straighten her out and she got angry. We kissed and made up before bedtime.

- Typical MI questions: How long have you lived here? Can I have a ride to church in the morning? I am a good friend all day, including kissing good night as she went to her room.

- [Mrs. Y] seemed to be in a non-MI mode this am. Sometimes hard to tell as life goes on normally a lot of the time and I only discover later I was just a friend.

\section{Discussion}

The development of DM frequently interferes with care during the course of MaND. Borsje and colleagues report DM as one of the most common neuropsychiatric symptoms in communitydwelling persons with dementia. ${ }^{18}$ Misidentification of a caregiver sets the stage for disruptive and even dangerous consequences. An adult approached by a presumed imposter is unlikely to cooperate with personal care such as bathing, toileting, or dressing. Confusion, modesty, fear, or anger can lead a person with dementia to reject care, argue, flee, or even attack. The relationship between DM and aggressive behavior in persons with major neurocognitive disorder has been well-described. ${ }^{1,19}$ No medication reliably improves recognition, and no behavioral intervention has previously been shown to improve or correct recognition of a spouse.

Overall, participation in the non-pharmacologic intervention described here was associated with improved recognition. MDQ scores were reduced for both care recipients. Some DM persisted for both care recipients. The MDQ's apparent underestimation in care recipient 1 reinforced our view that valid measurement of DM change is best captured by using multiple measures: MDQ, NPI, as well as daily diary to capture the extent and manifestations of DM in daily life provided cues to potential triggers. Care recipient 1 responded to the personalized non-pharmacological intervention. The twice-daily review of images was associated with specific and nonspecific benefits. Not only was recognition of his caregiving wife improved, but in addition he improved on symptoms of delusion, hallucination, and agitation in frequency and severity. Furthermore, his wife's caregiving burden was reduced as measured by the NPI's stress associated with symptoms. Care recipient 2 also responded well, as seen by the change in emotional response to facial recognition assessment. 
We believe that this intervention succeeded in helping our two care dyads significantly by strengthening the relationship between memory and its associated affect. By repeatedly presenting images of familiar faces with music for positive reminiscence, the intervention enhances the connection between the faces and a positive emotional response. This may lead to less suspicion and fewer negative emotions when interacting with the individuals. Technological innovations such as the one studied may have a significant role in enhancing a sense of comfort and familiarity in persons with dementia.

Finally, this intervention introduces a pleasant interaction into the daily life of care recipient and caregiver, one which does not involve conflict or control struggles. With progression of cognitive impairment, increasing caregiver time is spent coaxing a care recipient through activities of daily living and this intervention incorporates the sharing of pleasant experiences and gentle encouragement. Further study of this intervention is in progress.

\section{Take-Home Message}

Many persons living with dementia and the families caring for them strive to continue homebased care for as long as that remains tolerable and safe. DM is an important barrier to continued care at home because it undermines the trust and comfort required for successful delivery of personal care and collaborative interactions with a caregiver. In the context of DM, the person with MaND's effort to resist care, to flee or even to attack a caregiver becomes understandable and, from his or her standpoint, justifiable. Although response of DM to medications is often disappointing, the success of this simple, digital, non-pharmacologic intervention holds out hope for effectively managing the delusions that would otherwise shorten the duration of successful home-based care.

\section{Acknowledgements}

The research described in this article was supported in part by the Rotary Club.

Correspondence: Anne Adams, SimpleC LLC, research@simplec.com

\section{References}

1. Silva, J. A., Leong, G. B., Weinstock, R., \& Ruiz-Sweeney, M. (2001, May). Delusional misidentification and aggression in Alzheimer's disease. Journal of Forensic Sciences, 46(3), 581-585. PubMed

2. American Psychiatric Association. (2013). Diagnostic and statistical manual of mental disorders, $5^{\text {th }}$ ed. Arlington, VA: American Psychiatric Association

3. Perini, G., Carlini, A., Pomati, S., Alberoni, M., Mariani, C., Nemni, R., \& Farina, E. (2016, October-December). Misidentification delusions: Prevalence in different types of dementia and validation of a structured questionnaire. Alzheimer Disease and Associated Disorders, 30(4), 331-337. PubMed https://doi.org/10.1097/WAD.0000000000000141

4. Darby, R. R., Laganiere, S., Pascual-Leone, A., Prasad, S., \& Fox, M. D. (2017, February). Finding the imposter: Brain connectivity of lesions causing delusional misidentifications. Brain, 140(2), 497-507. PubMed https://doi.org/10.1093/brain/aww288 
5. Douglas, S., James, I., \& Ballard, C. (2004). Non-pharmacological interventions in dementia. Advances in Psychiatric Treatment, 10(3), 171-177. https://doi.org/10.1192/apt.10.3.171

6. Kerssens, C., Sattler, M., \& Monteiro, A. (2014, July). Managing dementia symptoms and needs using technology. Journal of Gerontological Nursing, 40(7), 16-20. PubMed https://doi.org/10.3928/00989134-20140513-01

7. Kerssens, C., Kumar, R., Adams, A. E., Knott, C. C., Matalenas, L., Sanford, J. A., \& Rogers, W. A. (2015, February). Personalized technology to support older adults with and without cognitive impairment living at home. American Journal of Alzheimer's Disease and Other Dementias, 30(1), 85-97. PubMed https://doi.org/10.1177/1533317514568338

8. Astell, A. J., Bouranis, N., Hoey, J., Lindauer, A., Mihailidis, A., Nugent, C., \& Robillard, J. M., \& the Technology and Dementia Professional Interest Area .... (2019). Technology and dementia: The future is now. Dementia and Geriatric Cognitive Disorders, 47(3), 131-139. PubMed https://doi.org/10.1159/000497800

9. Tyack, C., \& Camic, P. M. (2017, August). Touchscreen interventions and the well-being of people with dementia and caregivers: A systematic review. International Psychogeriatrics, 29(8), 1261-1280. PubMed https://doi.org/10.1017/S1041610217000667

10. Folstein, M. F., Folstein, S. E., \& McHugh, P. R. (1975, November). "Mini-mental state”. A practical method for grading the cognitive state of patients for the clinician. Journal of Psychiatric Research, 12(3), 189-198. PubMed https://doi.org/10.1016/00223956(75)90026-6

11. Nasreddine, Z. S., Phillips, N. A., Bédirian, V., Charbonneau, S., Whitehead, V., Collin, I., . . . Chertkow, H. (2005, April). The Montreal Cognitive Assessment, MoCA: A brief screening tool for mild cognitive impairment. Journal of the American Geriatrics Society, 53(4), 695-699. PubMed https://doi.org/10.1111/j.1532-5415.2005.53221.x

12. Yesavage, J. A., Brink, T. L., Rose, T. L., Lum, O., Huang, V., Adey, M., \& Leirer, V. O. (1982). Development and validation of a geriatric depression screening scale: A preliminary report. Journal of Psychiatric Research, 17(1), 37-49. PubMed https://doi.org/10.1016/0022-3956(82)90033-4

13. Cummings, J. L. (1997, May). The Neuropsychiatric Inventory: Assessing psychopathology in dementia patients. Neurology, 48(5, Suppl 6), 10S-16S. PubMed https://doi.org/10.1212/WNL.48.5_Suppl_6.10S

14. Farina, E., \& Pomati, S. (2016). Misidentification delusion questionnaire (MDQ).

15. Zarit, S. H., Reever, K. E., \& Bach-Peterson, J. (1980, December). Relatives of the impaired elderly: Correlates of feelings of burden. The Gerontologist, 20(6), 649-655. PubMed https://doi.org/10.1093/geront/20.6.649

16. Logsdon, R. G., Gibbons, L. E., McCurry, S. M., \& Teri, L. (1999). Quality of life in Alzheimer's disease: Patient and caregiver reports. Journal of Mental Health and Aging, $5(1), 21-32$. 
Doi: $10.32481 /$ djph.2021.09.007

17. Roediger, H. L., III, \& Karpicke, J. D. (2006, September). The power of testing memory: Basic research and implications for educational practice. Perspect Psychol Sci, 1(3), 181210. PubMed https://doi.org/10.1111/j.1745-6916.2006.00012.x

18. Borsje, P., Wetzels, R. B., Lucassen, P. L., Pot, A. M., \& Koopmans, R. T. (2015, March). The course of neuropsychiatric symptoms in community-dwelling patients with dementia: A systematic review. International Psychogeriatrics, 27(3), 385-405. PubMed https://doi.org/10.1017/S1041610214002282

19. Kaufman, K. R., Newman, N. B., \& Dawood, A. (2014, August). Capgras delusion with violent behavior in Alzheimer dementia: Case analysis with literature review. Ann Clin Psychiatry, 26(3), 187-191. PubMed

Copyright (c) 2021 Delaware Academy of Medicine / Delaware Public Health Association.

This is an Open Access article distributed under the terms of the Creative Commons Attribution Non-Commercial License (https://creativecommons.org/licenses/by-nc-nd/4.0/) which permits unrestricted non-commercial use, distribution, and reproduction in any medium, provided the original work is properly cited. 